\title{
Strategy-proof voting rules on a multidimensional policy space for a continuum of voters with elliptic preferences
}

\author{
Hans Peters • Souvik Roy • Ton Storcken
}

Received: 27 November 2010 / Accepted: 9 February 2011 / Published online: 24 February 2011 (C) The Author(s) 2011. This article is published with open access at SpringerLink.com

\begin{abstract}
We consider voting rules on a multidimensional policy space for a continuum of voters with elliptic preferences. Assuming continuity, $\gamma$-strategy-proofnessmeaning that coalitions of size smaller or equal to a small number $\gamma$ cannot manipulate-and unanimity, we show that such rules are decomposable into one-dimensional rules. Requiring, additionally, anonymity leads to an impossibility result. The paper can be seen as an extension of the model of Border and Jordan (1983) to a continuum of voters. Contrary, however, to their finite case where single voters are atoms, in our model with nonatomic voters even a small amount of strategy-proofness leads to an impossibility.
\end{abstract}

Keywords Strategy-proof voting - Continuum of voters .

Multidimensional policy space $\cdot$ Elliptic preferences

JEL Classification D71 $\cdot$ D72

We thank an anonymous referee for helpful comments.

H. Peters $(\bowtie) \cdot T$. Storcken

Department of Quantitative Economics, Maastricht University, Maastricht, The Netherlands

e-mail: H.Peters@maastrichtuniversity.nl

\section{T. Storcken}

e-mail: T.Storcken@maastrichtuniversity.nl

S. Roy

Department of Economics, University of Caen, Caen, France

e-mail: souvik.roy@unicaen.nl 


\section{Introduction}

We consider voting rules for situations with a large number of voters, who have singlepeaked preferences on a multidimensional policy space. We assume that the voters actually constitute a continuum, more precisely, they are elements of a nonatomic measure space. This is a good approximation of a situation with many voters, such as for instance national elections, and it makes it possible to accommodate the fact that in such situations small coalitions have no or only little influence. The policy space is represented by the hypercube $[0,1]^{k}$. A point in this hypercube represents a position with respect to $k$ different issues or criteria. In the context of a national election, such a point may also represent a specific political party; in this context, voters normally vote for a finite number of parties, but allowing them to vote for any position in the hypercube is again an approximation of the finite party case. See Maus et al. (2006) for more discussion on this issue.

Single-peakedness of preferences means that each voter has an ideal point, and preference decreases when moving away from this point. Specifically, we assume that preferences are separable quadratic, i.e., have elliptic indifference curves: this leaves sufficient room to model trade-offs between policies (criteria, coordinates) while potentially allowing for reasonable voting rules.

Our model extends the model of Border and Jordan (1983), where the number of voters is finite, to a continuum of voters. ${ }^{1}$ Border and Jordan impose strategy-proofness and unanimity on a voting rule and obtain decomposability: such a voting rule is completely determined by one-dimensional voting rules applied to each coordinate separately, and these one-dimensional voting rules are of the type as characterized earlier in Moulin (1980). It is well-known that, although these one-dimensional voting rules are group-strategy proof (cannot be manipulated by coalitions of voters) this property is lost as soon as the dimension is higher than one. See, recently, Barberà et al. (2010).

In our model, manipulation by single voters is not possible since single voters do not have any influence. Instead, we impose that coalitions of positive but small size cannot manipulate, and call this condition $\gamma$-strategy-proofness, where $\gamma$ is a small but positive number. We also impose unanimity and a weak continuity condition (based, technically, on convergence in measure). Like Border and Jordan (1983) we obtain decomposability of the rule into one-dimensional rules. Under the additional condition of anonymity, these one-dimensional rules are those characterized by Maus et al. (2006). However, any composition of such rules for higher dimensions fails to be $\gamma$-strategy-proof for any $\gamma>0$. Thus, we obtain an impossibility result: there is no unanimous, anonymous and continuous rule for more than one dimension which is non-manipulable, even if we require this only for coalitions of arbitrarily small size.

These results can be considered to be in line with what is known in the literature. We obtain decomposability just as Border and Jordan (1983), but contrary to them we obtain from this an impossibility result since, basically, our strategy-proofness condition is one of group strategy-proofness. Thus, and in contrast to Border and Jordan, our

\footnotetext{
1 We only mention the directly relevant literature here. For an extensive survey, see Barberà (2010).
} 
main conclusion is that in situations with a large number of voters there is no scope for even a weak form of strategy-proofness under reasonable additional conditions. From a technical point of view, although our proofs share elements with proofs in Border and Jordan (1983), they are nevertheless considerably different, due to the possibility of coalitions of any arbitrary size instead of a finite number of 'atomic' players.

In Sect. 2 the model is introduced, Sect. 3 deals with the one-dimensional case, and Sect. 4 presents the mentioned decomposability and impossibility results. Section 5 contains a few concluding remarks about the tightness of the imposed conditions.

\section{Preliminaries}

Let $(\Omega, \Sigma, \lambda)$ be a nonatomic measure space. Every $t \in \Omega$ is a voter and every element $S$ of the $\sigma$-field $\Sigma$ is a coalition. The nonnegative number $\lambda(S)$ is interpreted as the size of $S$. We assume that $\Omega$ has positive measure and normalize its size to one: $\lambda(\Omega)=1$.

The set of alternatives is the hypercube $A=[0,1]^{k}$, where $k \in \mathbb{N}$. Let $\mathcal{Q}$ denote the set of all elliptic preferences on $A$, i.e., binary relations representable by a utility function of the form $x \mapsto-\sum_{j=1}^{k} w_{j}\left(x_{j}-p_{j}\right)^{2}, x=\left(x_{1}, \ldots, x_{k}\right) \in A$, for some peak $p=\left(p_{1}, \ldots, p_{k}\right) \in A$ and vector of positive weights $w \in W$, where $W=\left\{\left(w_{1}, \ldots, w_{k}\right) \in \mathbb{R}_{++}^{k} \mid \max \left\{w_{1}, \ldots, w_{k}\right\}=1\right\}$. Such a preference can be identified with the pair $(p, w) \in A \times W$, and $\mathcal{Q}$ with the set $A \times W$. We endow $\mathcal{Q}=A \times W$ with the Borel $\sigma$-field.

A profile is a measurable function $R: \Omega \rightarrow \mathcal{Q}$. Thus, in a profile $R$ every voter $t$ is endowed with an elliptic preference $R(t) \in \mathcal{Q}$. The peak and weight vector of $R(t)$ are denoted by $p(R(t))$ and $w(R(t))$. The set of all profiles is denoted by $\rho$. Every $R \in \rho$ induces a (probability) measure $\lambda_{R}$ on $A$ by defining $\lambda_{R}(B)=\lambda(\{t \in \Omega \mid p(R(t)) \in$ $B\}$ ) for every measurable subset $B$ of $A$. This measure $\lambda_{R}$ represents the distribution of the peaks of the votes resulting from an election where every voter $t \in \Omega$ votes according to $R(t)$.

Let $J$ be some ordered index set. A collection of profiles $\left(R_{j}\right)_{j \in J}$ converges to a profile $R$ if $\left(\lambda\left(\left\{t \in \Omega \mid R_{j}(t) \neq R(t)\right\}\right)\right)_{j \in J}$ converges to $0 .^{2}$

A voting rule or briefly rule is a map $F: \rho \rightarrow A$.

A rule $F$ is anonymous if $F(R)=F(\dot{\widetilde{R}})$ for all $R, \widetilde{R} \in \rho$ such that $\lambda(\{t \in \Omega$ | $R(t) \in Q\})=\lambda\left(\left\{t \in \Omega \mid R^{\prime}(t) \in Q\right\}\right)$ for every measurable subset $Q$ of $\mathcal{Q}$.

A rule $F$ is unanimous if $F(R)=p$ for all $R \in \rho$ such that there is a $(p, w) \in \mathcal{Q}$ satisfying $R(t)=(p, w)$ for all $t \in \Omega$.

A rule $F$ is continuous if $\left(F\left(R_{j}\right)\right)_{j \in J}$ (where $J$ is an ordered index set) converges to $F(R)$ whenever the collection of profiles $\left(R_{j}\right)_{j \in J}$ converges to $R \in \rho$. Observe that continuity of a rule $F$ implies that coalitions of size zero and in particular single voters are powerless: that is, $F(R)=F(\widetilde{R})$ whenever $\lambda(\{t \in \Omega \mid R(t) \neq \widetilde{R}(t)\})=0$. This is a very weak continuity condition: it does not imply anything if each voter's preferences in two different profiles are very close.

\footnotetext{
2 This is convergence in measure.
} 


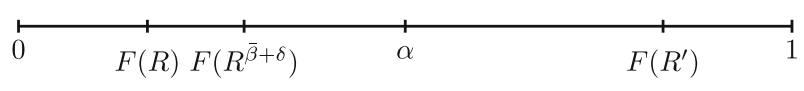

Fig. 1 Illustrating the proof of Lemma 1. The number $\alpha$ is the midpoint between $F(R)$ and $F\left(R^{\prime}\right)$. All voters in $S$ have their peaks to the right of $\alpha$

Let $\gamma \in \mathbb{R}, 0<\gamma \leq 1$. A rule $F$ is $\gamma$-manipulable by a coalition $S$ at a profile $R \in \rho$ if $\lambda(S) \leq \gamma$ and there is a profile $\widetilde{R} \in \rho$ with $\widetilde{R}(t)=R(t)$ for all $t \in \Omega \backslash S$ such that $F(\widetilde{R}) P(t) F(R)$ for all $t \in S$, where $P(t)$ denotes the asymmetric part of $R(t)$. A rule $F$ is $\gamma$-strategy-proof ( $\gamma$-SP) if it is not $\gamma$-manipulable by any coalition $S$ at any profile $R$.

\section{The one-dimensional case}

In the one-dimensional case $(k=1)$ preferences are completely determined by their peaks. Therefore, we can identify $\mathcal{Q}$ with the set $A=[0,1]$ and $\rho$ with the set $[0,1]^{\Omega}$. In this case, $\gamma$-SP implies that no coalition of any size can manipulate, as the following lemma shows. The proof of this lemma is illustrated in Fig. 1.

Lemma 1 Let $k=1$, let $0<\gamma \leq 1$, and let $F$ be continuous and $\gamma$-SP. Then $F$ is $\gamma^{\prime}$-SP for every $\gamma^{\prime} \in(0,1]$.

Proof Let $\gamma^{\prime} \in(0,1]$. If $\gamma^{\prime} \leq \gamma$ then clearly $F$ is $\gamma^{\prime}$-SP. Now assume $\gamma^{\prime}>\gamma$ and for contradiction suppose that $F$ is $\gamma^{\prime}$-manipulable by some coalition $S \in \Sigma$ at profile $R \in \rho$. Then $\gamma<\lambda(S) \leq \gamma^{\prime}$ and there is a profile $R^{\prime}$ with $R^{\prime}(t)=R(t)$ for all $t \in \Omega \backslash S$ such that $F\left(R^{\prime}\right) P(t) F(R)$ for all $t \in S$. Hence $F\left(R^{\prime}\right) \neq F(R)$, w.l.o.g. ${ }^{3}$ $F\left(R^{\prime}\right)>F(R)$. Then $p(R(t))>\alpha$ for all $t \in S$, where $\alpha:=\frac{1}{2}\left(F(R)+F\left(R^{\prime}\right)\right)$, since each $R(t)$ is a symmetric single-peaked preference on $A=[0,1]$.

For each $0 \leq \beta \leq \lambda(S)$ let $S^{\beta} \subseteq S$ with $\lambda\left(S^{\beta}\right)=\beta$ and such that $S^{\beta} \subseteq S^{\beta^{\prime}}$ whenever $\beta \leq \bar{\beta}^{\prime}$; this is possible since $\lambda$ is nonatomic. Let $R^{\beta}$ be defined by $R^{\bar{\beta}}(t)=$ $R^{\prime}(t)$ for $t \in S^{\beta}$ and $R^{\beta}(t)=R(t)$ for $t \in \Omega \backslash S^{\beta}$. Let $\bar{\beta}:=\max \{0 \leq \beta \leq \lambda(S) \mid$ $\left.F\left(R^{\beta}\right)=F(R)\right\}$. Then $\bar{\beta}$ is well-defined by continuity of $F, \bar{\beta}<\lambda(S)$ and $\bar{F}\left(R^{\beta}\right)>$ $F(R)=F\left(R^{\bar{\beta}}\right)$ for all $\beta>\bar{\beta}$. Again by continuity of $F$ there is a $\delta<\min \{\gamma, \lambda(S)-\bar{\beta}\}$ such that $F\left(R^{\bar{\beta}+\delta}\right)<\alpha$. This implies that the coalition $S^{\bar{\beta}+\delta} \backslash S^{\bar{\beta}} \gamma$-manipulates $F$ at $R^{\bar{\beta}}$, a contradiction.

We call a one-dimensional rule $F$ strategy-proof if it is $\gamma$-SP for every $\gamma \in(0,1]$. By Lemma 1 , every one-dimensional $\gamma$-SP rule $F$ is strategy-proof. In Maus et al. (2006) all continuous, unanimous, anonymous and strategy-proof one-dimensional rules have been characterized. ${ }^{4}$ In this characterization, a central role is played by the family $\mathcal{F}$ of all nondecreasing (i.e., weakly increasing) and continuous functions

\footnotetext{
3 Without loss of generality.

4 Maus et al. (2006) use the stronger property of Pareto optimality but it is easy to see that their characterization still holds with unanimity instead of Pareto optimality. The argument goes as follows. If $F$ is not Pareto optimal then there is a profile $R$ and an interval $[a, b] \subseteq[0,1]$ such that almost all peaks of $R$ are in $[a, b]$ but (say) $F(R)<a$. Gradually change the profile $R$ to a unanimous profile $Q$ with all peaks at $a$. By continuity and SP, $F(Q) \leq F(R)<a$, hence $F$ is not unanimous.
} 
$f:[0,1] \rightarrow A$ with $f(0)=0$ and $f(1)=1$. Let $f \in \mathcal{F}$. We define a rule $F^{f}$ associated with $f$, as follows. (See Fig. 2 later on for an illustration.) Let $R \in \rho$. Consider the induced decumulative distribution $D^{R}$ defined by $D^{R}(x):=\lambda_{R}([x, 1])$ for all $x \in A$. Now

$$
F^{f}(R):=\max \left\{x \in A \mid f\left(D^{R}(x)\right) \geq x\right\}
$$

The rule $F^{f}$ is well-defined since the set of which the maximum is taken is nonempty (it contains $x=0), f$ is continuous and $D^{R}$ is left-continuous. The mentioned characterization is as follows.

Theorem 1 Let $k=1$. A rule $F$ is continuous, unanimous, strategy-proof and anonymous if and only if there is an $f \in \mathcal{F}$ such that $F=F^{f}$.

Theorem 1 and Lemma 1 imply the following result.

Corollary 1 Let $k=1$ and $0<\gamma \leq 1$. A rule $F$ is continuous, unanimous, $\gamma$-SP and anonymous if and only if there is an $f \in \mathcal{F}$ such that $F=F^{f}$.

\section{The general case: decomposability and impossibility}

The main result of this section and of the paper is the following decomposability theorem.

Theorem 2 Let $0<\gamma \leq 1$. If a rule $F$ is continuous, unanimous, and $\gamma$-SP then for each $j=1, \ldots, k$ there is a continuous, unanimous and $\gamma-S P$ rule $F^{j}:[0,1]^{\Omega} \rightarrow$ $[0,1]$ such that for all $R=(p(t), w(t))_{t \in \Omega} \in \rho$,

$$
F_{j}(R)=F^{j}\left(\left(p_{j}(t)\right)_{t \in \Omega}\right) \text { for each } j=1, \ldots, k
$$

where $F_{j}(R)$ is the $j$-th coordinate of $F(R)$.

Proof See Appendix.

Theorem 2 implies in particular that a rule with the mentioned properties is peaksonly, i.e., depends only on the peaks and not on the weight vectors of the preferences. Unfortunately, requiring, in addition, anonymity leads to an impossibility, as the following result shows.

Theorem 3 Let $0<\gamma \leq 1$ and let $k \geq 2$. There is no rule $F$ that is continuous, unanimous, anonymous, and $\gamma-S P$.

Proof Suppose $F$ is a continuous, unanimous, anonymous, and $\gamma$-SP rule. Then by Theorem 2 and Corollary 1 , there are $f_{j} \in \mathcal{F}, j=1, \ldots, k$, such that for all $R=(p(t), w(t))_{t \in \Omega} \in \rho$,

$$
F_{j}(R)=F^{f_{j}}\left(\left(p_{j}(t)\right)_{t \in \Omega}\right) \quad \text { for each } j=1, \ldots, k \text {. }
$$



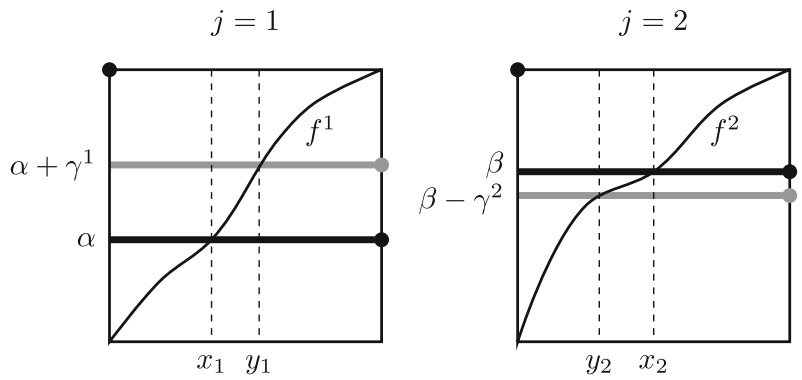

Fig. 2 Illustrating the proof of Theorem 3. In the left diagram, the thick black curve represents the decumulative distribution of $\lambda^{1}$, and the grey curve the distribution resulting from manipulation by $T_{00} \cup T_{11}$. The right diagram illustrates the situation for the second coordinate, with similar explanation.

We show, for contradiction, that a rule $F$ given by (2) is not $\gamma$-SP. We assume $k=2$, the construction that follows can easily be embedded in higher dimensions.

First, choose numbers $\alpha, \beta$, and $\varepsilon$ such that

- $0<\alpha<\alpha+\varepsilon<1$ and $0<\beta-\varepsilon<\beta<1$

- $f^{1}(\xi)>f^{1}(\alpha)$ for all $\xi \in(\alpha, \alpha+\varepsilon)$

- $f^{2}(\xi)<f^{2}(\beta)$ for all $\xi \in(\beta-\varepsilon, \beta)$.

This is possible since $f^{1}, f^{2} \in \mathcal{F}$, i.e., $f^{1}:[0,1] \rightarrow[0,1]$ and $f^{2}:[0,1] \rightarrow$ $[0,1]$ are nondecreasing and continuous functions with $f^{1}(0)=f^{2}(0)=0$ and $f^{1}(1)=f^{2}(1)=1$. Let $\delta$ be a number such that $0<\delta<1$ and $1-\alpha-\beta<\delta<$ $\min \{1-\alpha, 1-\beta\}$. Let $\Omega$ be partitioned into coalitions $S_{00}, S_{10}, S_{01}$, and $S_{11}$ such that $\lambda\left(S_{00}\right)=\delta, \lambda\left(S_{10}\right)=1-\beta-\delta, \lambda\left(S_{01}\right)=1-\alpha-\delta$, and $\lambda\left(S_{11}\right)=\delta+\alpha+\beta-1$. Consider a profile $R$ such that

$$
p(R(t))=\left\{\begin{array}{ll}
(0,0) & \text { if } t \in S_{00} \\
(1,0) & \text { if } t \in S_{10} \\
(0,1) & \text { if } t \in S_{01} \\
(0,0) & \text { if } t \in S_{00}
\end{array} \quad \text { for all } t \in \Omega\right.
$$

Some of the weight vectors are chosen later in the proof, but they do not influence $F(R)$, since $F$ is peaks-only.

Let $\lambda^{1}$ denote the marginal probability measure on the set of first coordinates $[0,1]$ induced by $R$. Then $\lambda^{1}(0)=1-\alpha$ and $\lambda(1)=\alpha$. Therefore, $F^{f^{1}}\left(\left(p_{1}(R(t))\right)_{t \in \Omega}\right)=$ $f^{1}(\alpha)$. Similarly we derive $F^{f^{2}}\left(\left(p_{2}(R(t))\right)_{t \in \Omega}\right)=f^{2}(\beta)$. Write $x_{1}=f^{1}(\alpha)$ and $x_{2}=f^{2}(\beta)$, then $F(R)=\left(x_{1}, x_{2}\right)$. See Fig. 2 for an illustration.

We now show that $F$ can be $\gamma$-manipulated at $R$. Let $0<\gamma^{1}, \gamma^{2}<\varepsilon$ such that $\gamma^{1}+\gamma^{2} \leq \gamma, \gamma^{1}<\delta=\lambda\left(S_{00}\right)$, and $\gamma^{2}<\delta+\alpha+\beta-1=\lambda\left(S_{11}\right)$. Let $T_{00} \subseteq S_{00}$ with $\lambda\left(T_{00}\right)=\gamma^{1}$ and let $T_{11} \subseteq S_{11}$ with $\lambda\left(T_{11}\right)=\gamma^{2}$. Now consider a profile $R^{\prime}$ with $p\left(R^{\prime}(t)\right)=(1,0)$ for all $t \in T_{00} \cup T_{11}$, and with $p\left(R^{\prime}(t)\right)=p(R(t))$ for all other $t$. Then $F\left(R^{\prime}\right)=\left(y_{1}, y_{2}\right)$ where $y_{1}=f^{1}\left(\alpha+\gamma^{1}\right)>x_{1}$ and $y_{2}=f^{2}\left(\beta-\gamma^{2}\right)<y^{2}$. Now it is straightforward to find a weight vector for the voters in $T_{00}$ such that $y P(t) x$ for all $t \in T_{00}$, and a weight vector for the voters in $T_{11}$ such that $y P(t) x$ for all $t \in T_{11}$; this boils down to constructing two ellipses with centers at $(0,0)$ and at $(1,1)$ 
respectively, both crossing through $x$ and having $y$ in the interior. But this means that $T_{00} \cup T_{11}$ can $\gamma$-manipulate $F$ at $R$ by changing their preferences to $R^{\prime}$.

\section{Concluding remarks}

How tight are the decomposability (Theorem 2) and impossibility (Theorem 3) results?

\subsection{Without unanimity}

Under $(\gamma$-)strategy-proofness, unanimity is equivalent to the requirement that the voting rule $F$ be surjective, i.e., its range is $[0,1]^{k}$. Without this condition, Theorem 2 no longer holds: in the following example we construct a voting rule which is $(\gamma$-)strategy-proof and continuous but not decomposable.

Example 1 Let $k=2$, and for every preference $(p, w)$ let $p^{\prime} \in D:=\left\{x \in[0,1]^{2} \mid\right.$ $\left.x_{1}=x_{2}\right\}$ be the maximally preferred point of $D$, i.e., $w_{1}\left(p_{1}^{\prime}-p_{1}\right)^{2}+w_{2}\left(p_{2}^{\prime}-p_{2}\right)^{2} \leq$ $w_{1}\left(x_{1}-p_{1}\right)^{2}+w_{2}\left(x_{2}-p_{2}\right)^{2}$ for all $x \in D$. Consider the set of symmetric single-peaked preferences on $D$, represented by their peaks, and let $G$ be a continuous, unanimous, strategy-proof and anonymous one-dimensional voting rule on $D$ as in Theorem 1 ( $D$ can be identified with the interval $[0,1])$. For a profile $R: \Omega \rightarrow[0,1]^{2} \times W$ with $R(t)=(p(t), w(t))$ for each $t \in \Omega$ we define $F(R)=G\left(\left(p(t)^{\prime}\right)_{t \in \Omega}\right) \in D$. Then it is not difficult to check that the voting rule $F$, defined in this way, is $(\gamma$-)strategy-proof and continuous. Clearly, it is not decomposable in the sense of Theorem 2: $F(R)$ depends not only on the peaks but also on the weights of the preferences in $R$.

The basic characteristic of the construction in Example 1 is that for a preference $(p, w)$ with $p$ not in the range ( $D$ in the example) the most preferred point of the range depends not only on $p$ but also on the weights $w$. One can show, more generally, that if this can happen then a voting rule with that range (but full domain) which is continuous and $(\gamma$-)strategy-proof cannot be decomposable. This leads to the conjecture that such a rule is decomposable exactly when the range is a hypercube (hence, rectangular in the two-dimensional case).$^{5}$ Obvious examples are the constant voting rule, or a rule with range $\left\{x \in[0,1]^{k} \mid \exists j \forall \ell \neq j\left[x_{\ell}=0\right]\right\}$. Without continuity, the range can be Cartesian without being a hypercube. As an example of the latter, consider the rule, for $k=2$, with range $\{(0,0),(1,0),(1,1),(0,1)\}$, obtained by using majority voting plus an appropriate tie-breaking rule to decide between up or down and left or right.

Also for the case of finitely many voters, the exact range condition guaranteeing decomposability under (only) strategy-proofness is, to the best of our knowledge, an open problem [already mentioned in Border and Jordan (1983)]. For the onedimensional case, all rules satisfying strategy-proofness, anonymity, and continuity are described in Remark 3.7 in Maus et al. (2006).

\footnotetext{
5 Results like this are reported for the case of finitely many voters in for instance Sprumont (1995) and Weymark (1999).
} 


\subsection{Without anonymity}

We conjecture that dropping anonymity is not going to change Theorem 3 in an essential way and may only lead to some kind of 'invisible dictator' result, as in Kirman and Sondermann (1972). Dropping anonymity in the one-dimensional case (Theorem 1) would imply that a rule is no longer characterized by a function $f \in \mathcal{F}$ (cf. Section 3) but by some map $\varphi: \Sigma \rightarrow[0,1]$, satisfying certain conditions (among which are continuity and monotonicity) and with $\varphi(S)$ representing the outcome of the voting rule when coalition $S$ votes for 0 while $\Omega \backslash S$ votes for 1 .

\subsection{Without continuity}

Interestingly, dropping continuity allows for rules which perhaps do not offer real compromises but at least are not dictatorial in any way. An example of such a rule is to choose the left or right endpoint of the support of a profile on each coordinate separately by using majority voting plus an appropriate tie-breaking rule.

\subsection{Different domains}

Finally, changing and in particular enlarging the domain of preferences will in general only make it more difficult to find strategy-proof voting rules, cf. Zhou (1991) for the case of finitely many voters.

\section{Appendix: Proof of Theorem 2}

We recall that the support of a probability measure $\mu$ on $A$ is the set of all points in $A$ such that every open neighborhood of such a point has positive measure. Equivalently, it is the smallest (in terms of set inclusion) closed subset that has measure 1. We denote the support of $\mu$ by $\operatorname{supp}(\mu)$ and for $j=1, \ldots, k$ we define the set

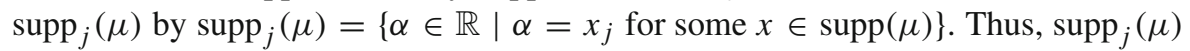
is the projection of $\operatorname{supp}(\mu)$ on the $j$-th coordinate.

For a subset $X$ of some Euclidean space we denote by conv $(X)$ the convex hull of $X$, i.e., the smallest convex set containing $X$. The first lemma shows that a continuous, unanimous and $\gamma$-SP rule assigns to each profile a point each coordinate of which is in the projection on that coordinate of the support of the probability measure generated by the profile.

Lemma 2 Let $0<\gamma \leq 1$. Let $F$ be continuous, unanimous and $\gamma-S P$, and let $R \in \rho$. Then $F_{j}(R) \in \operatorname{conv}\left(\operatorname{supp}_{j}\left(\lambda_{R}\right)\right)$ for each $j=1, \ldots, k$.

Proof (a) First, suppose that there is an $\alpha>0$ such that $w_{j}(t)>\alpha$ for all $j=1, \ldots, k$ and all $t \in \Omega$, where $R(t)=(p(t), w(t))$. Suppose, for a contradiction, that $F_{1}(R)<$ $r_{1}$ where $\left[r_{1}, r_{2}\right]=\operatorname{conv}\left(\operatorname{supp}_{1}\left(\lambda_{R}\right)\right)$. Let $p:=\left(\frac{1}{2}\left(r_{1}+F_{1}(R)\right), F_{2}(R), \ldots, F_{k}(R)\right) \in$ 
$A$ and for each $\delta>0$ let $w(\delta)=(\delta, 1, \ldots, 1) \in W$. Let

$$
E(\delta):=\left\{x \in A \mid \sum_{j=1}^{k} w_{j}(\delta)\left(x_{j}-p_{j}\right)^{2} \leq \sum_{j=1}^{k} w_{j}(\delta)\left(F_{j}(R)-p_{j}\right)^{2}\right\}
$$

denote the set of points weakly preferred to $F(R)$ by a voter with preference $(p, w(\delta))$. We first prove the following claim.

Claim. Let $0<\varepsilon<\frac{1}{2}\left(r_{1}-F_{1}(R)\right)$. Then there is a $\delta>0$ such that for all $x \in E(\delta)$ with $x_{1}+\varepsilon<p_{1}$ and all $y \in E(\delta)$ with $y_{1}=x_{1}+\varepsilon$, we have $y P(t) x$ for all $t \in \Omega$.

Proof. We have to show that there is a $\delta>0$ such that for all $t \in \Omega$ and all $x$ and $y$ as in the claim the following inequality holds:

$$
w_{1}\left(\left(x_{1}+\varepsilon\right)-q_{1}\right)^{2}+\sum_{j=2}^{k} w_{j}\left(y_{j}-q_{j}\right)^{2}<w_{1}\left(x_{1}-q_{1}\right)^{2}+\sum_{j=2}^{k} w_{j}\left(x_{j}-q_{j}\right)^{2},
$$

where $(w, q)=R(t)$. This inequality is equivalent to

$$
w_{1}\left[\left(\left(x_{1}+\varepsilon\right)-q_{1}\right)^{2}-\left(x_{1}-q_{1}\right)^{2}\right]<\sum_{j=2}^{k} w_{j}\left[\left(x_{j}-q_{j}\right)^{2}-\left(y_{j}-q_{j}\right)^{2}\right] .
$$

Since $x, y \in A(\delta)$, the absolute value of the expression at the right-hand side of this inequality can be made uniformly small (that is, for all $(q, w) \in \mathcal{Q})$ by choosing $\delta$ small. The expression at the left-hand side is negative but bounded away from zero:

$$
\begin{aligned}
w_{1}\left[\left(\left(x_{1}+\varepsilon\right)-q_{1}\right)^{2}-\left(x_{1}-q_{1}\right)^{2}\right] & =w_{1}\left[\left(q_{1}-x_{1}-\varepsilon\right)^{2}-\left(q_{1}-x_{1}\right)^{2}\right] \\
& =-\varepsilon w_{1}\left[2\left(q_{1}-x_{1}\right)-\varepsilon\right] \\
& <-\varepsilon w_{1}\left[2\left(r_{1}-p_{1}\right)+\varepsilon\right] \\
& <-\varepsilon \omega\left[2\left(r_{1}-p_{1}\right)+\varepsilon\right] .
\end{aligned}
$$

This concludes the proof of the Claim.

Let $m$ be the smallest integer larger than $1 / \gamma$, let $\varepsilon<\left(p_{1}-F_{1}(R)\right) / m$, and take $\delta$ as in the Claim. Take $S^{1}, \ldots, S^{m} \in \Sigma$ pairwise disjoint with $\lambda\left(S^{i}\right)=\gamma$ for $i=1, \ldots, m-1$ and $\lambda\left(S^{m}\right)=1-(m-1) \gamma \leq \gamma$. Let $S^{0}=\emptyset$. For $i=0, \ldots, m$ define $R^{i} \in \rho$ by $R^{i}(t)=(p, w(\delta))$ for all $t \in \cup_{\ell=0}^{i} S^{\ell}$ and $R^{i}(t)=R(t)$ for all remaining $t$. By $\gamma$-SP, $F\left(R^{1}\right) \in E(\delta)$ otherwise $S^{1}$ can $\gamma$-manipulate $F$ at $R^{1}$. By the choice of $\delta$ as in the Claim, we have $F_{1}\left(R^{1}\right)<F_{1}(R)+\varepsilon$ otherwise $S_{1}$ can $\gamma$-manipulate $F$ at $R^{0}=R$. Again by $\gamma$-SP and the fact that $F\left(R^{1}\right) \in E(\delta)$, we have $F\left(R^{2}\right) \in E(\delta)$ otherwise $S^{2}$ can $\gamma$-manipulate $F$ at $R^{2}$. Again by the choice of $\delta$ as in the Claim, we have $F_{1}\left(R^{2}\right)<F_{1}\left(R^{1}\right)+\varepsilon<F_{1}(R)+2 \varepsilon$ otherwise $S_{2}$ can $\gamma$-manipulate $F$ at $R^{1}$. By performing this step $m$ times in total we obtain for the profile $R^{m}$ that $F_{1}\left(R^{m}\right)<F_{1}(R)+m \varepsilon<p_{1}$. By unanimity, however, $F\left(R^{m}\right)=p$, a contradiction. 
(b) Now let $R \in \rho$ be arbitrary. For $w \in W$ and $n \in \mathbb{N}$ define $w^{n} \in W$ by $w_{j}^{n}=w_{j}$ if $w_{j} \geq 1 / n$ and $w_{j}^{n}=1 / n$ if $w_{j}<1 / n$, for all $j=1, \ldots, k$. For each $n \in \mathbb{N}$ define $R^{n} \in \rho$ by $R^{n}(t)=\left(p(t), w^{n}(t)\right)$ if $R(t)=(p(t), w(t))$, for all $t \in \Omega$. We claim that $R^{n} \rightarrow R$ for $n \rightarrow \infty$. If not, then let $S^{1} \supseteq S^{2} \supseteq \ldots$ be defined by $S^{n}=\left\{t \in \Omega \mid R^{n}(t) \neq R(t)\right\}$ for all $n$. Then there is a $\delta>0$ such that $\lambda\left(\cap_{n \in \mathbb{N}} S^{n}\right) \geq \delta$, so that in particular $\cap_{n \in \mathbb{N}} S^{n} \neq \emptyset$. Take $t \in \cap_{n \in \mathbb{N}} S^{n}$ and let $R(t)=\left(p(t), w(t)\right.$, then there is some $j$ with $w_{j}(t)=0$, a contradiction since $w(t) \in W$.

By part (a) of the proof, $F_{j}\left(R^{n}\right) \in \operatorname{conv}\left(\operatorname{supp}_{j}\left(\lambda_{R^{n}}\right)\right)=\operatorname{conv}\left(\operatorname{supp}_{j}\left(\lambda_{R}\right)\right)$ for each $j=1, \ldots, k$ and all $n \in \mathbb{N}$. By continuity of $F$, this implies $F_{j}(R) \in$ $\operatorname{conv}\left(\operatorname{supp}_{j}\left(\lambda_{R}\right)\right)$ for each $j=1, \ldots, k$.

The following lemma shows that a continuous, unanimous, and $\gamma$-SP rule is peaksonly when restricted to profiles that live on only one coordinate.

Lemma 3 Let $0<\gamma \leq 1$ and let $F$ be continuous, unanimous, and $\gamma$-SP. Let $R=(p(t), w(t))_{t \in \Omega}, R^{\prime}=\left(p(t), w^{\prime}(t)\right)_{t \in \Omega} \in \rho$ and $j \in\{1, \ldots, k\}$ be such that $p_{\ell}(t)=p_{\ell}\left(t^{\prime}\right)$ for all $\ell \neq j$ and $t, t^{\prime} \in \Omega$. Then $F(R)=F\left(R^{\prime}\right)$.

Proof W.l.o.g. let $j=1$. Take $\varepsilon>0$ and let $S=\left\{t \in \Omega \mid p_{1}(t)<F_{1}(R)-\varepsilon\right\}$. We prove that $F(Q)=F(R)$ for the profile $Q$ with $Q(t)=\left(p(t), w^{\prime}(t)\right)$ for all $t \in S$ and $Q(t)=(p(t), w(t))$ for all $t \in \Omega \backslash S$. Then, since $\varepsilon>0$ is arbitrary, it follows by continuity of $F$ that $F\left(R^{\prime \prime}\right)=F(R)$ for the profile $R^{\prime \prime}$ with $R^{\prime \prime}(t)=(p(t), w \prime(t))$ for all $t$ with $p_{1}(t) \leq F_{1}(R)$ and $R^{\prime \prime}(t)=(p(t), w(t))$ for all other $t$. By repeating the argument for voters $t$ with $p_{1}(t)>F_{1}(R)$, we obtain $F\left(R^{\prime}\right)=F(R)$ and the proof is complete.

We are done if $\lambda(S)=0$. Now assume $\lambda(S)=\bar{\beta}>0$, and for each $0 \leq \beta \leq \bar{\beta}$ let $S^{\beta} \subseteq \Omega$ such that (i) $\lambda\left(S^{\beta}\right)=\beta$ and (ii) $S^{\beta} \subseteq S^{\beta^{\prime}}$ whenever $\beta \leq \beta^{\prime}$ (this is possible since $\lambda$ is nonatomic). For each $0 \leq \beta \leq \bar{\beta}$ define the profile $R^{\beta}$ by $R^{\beta}(t)=\left(p(t), w^{\prime}(t)\right)$ for all $t \in S^{\beta}$ and $R^{\beta}(t)=(p(t), w(t))$ for all $t \in \Omega \backslash S^{\beta}$. By Lemma $2, F_{\ell}\left(R^{\beta}\right)=F_{\ell}(R)$ for all $\ell \neq 1$. Let $\alpha=\max \left\{0 \leq \beta \leq \bar{\beta} \mid F_{1}\left(R^{\beta}\right)=\right.$ $\left.F_{1}(R)\right\}$. If $\alpha<\bar{\beta}$, take a number $\alpha^{\prime}$ with $\alpha<\alpha^{\prime} \leq \bar{\beta}, \alpha^{\prime} \leq \alpha+\gamma$, and such that $F_{1}\left(R^{\alpha^{\prime}}\right)>F_{1}(R)-\frac{1}{2} \varepsilon$ : this is possible by continuity of $F$. If $F_{1}\left(R^{\alpha^{\prime}}\right)<F_{1}(R)$ then coalition $S^{\alpha^{\prime}} \backslash S^{\alpha}$ can $\gamma$-manipulate $F$ at $R^{\alpha}$; if $F_{1}\left(R^{\alpha^{\prime}}\right)>F_{1}(R)$ then coalition $S^{\alpha^{\prime}} \backslash S^{\alpha}$ can $\gamma$-manipulate $F$ at $R^{\alpha^{\prime}}$. Hence, we have $\alpha=\bar{\beta}$, so $F(Q)=F\left(R^{\bar{\beta}}\right)=F(R)$.

The next lemma presents a technical result, which will be used in the proof of Lemma 5 below.

Lemma 4 Let $k \geq 2$ and $0<\varepsilon<1$. Then there is a $\delta>0$ such that for all $p, x, y \in A$ with either $p_{1} \leq y_{1} \leq x_{1}-\varepsilon$ or $p_{1} \geq y_{1} \geq x_{1}+\varepsilon$ we have $y P(t) x$, where $P(t)$ is the asymmetric part of $R(t)=(p,(1, \delta, \ldots, \delta)) \in \mathcal{Q}$.

Proof Take $\delta<\varepsilon^{2} /(k-1)$ and let $p, x, y \in A$ with $p_{1} \leq y_{1} \leq x_{1}-\varepsilon$ (the other case is analogous). Then $y P(t) x$ is equivalent to

$$
\delta\left(\sum_{j=2}^{k}\left(y_{j}-p_{j}\right)^{2}-\sum_{j=2}^{k}\left(x_{j}-p_{j}\right)^{2}\right)<\left(x_{1}-p_{1}\right)^{2}-\left(y_{1}-p_{1}\right)^{2} .
$$


The left-hand side of this equation is smaller than $\delta(k-1)$. For the right-hand side we have

$$
\begin{aligned}
\left(x_{1}-p_{1}\right)^{2}-\left(y_{1}-p_{1}\right)^{2} & \geq\left(y_{1}+\varepsilon-p_{1}\right)^{2}-\left(y_{1}-p_{1}\right)^{2} \\
& =\varepsilon\left(2 y_{1}-2 p_{1}+\varepsilon\right) \\
& \geq \varepsilon^{2} .
\end{aligned}
$$

Equation (3) now follows since $\delta(k-1)<\varepsilon^{2}$.

The next lemma is the basis for the proof of Theorem 2. It says that the $j$-th coordinate of the point assigned to a profile by a continuous, unanimous, and $\gamma$-SP rule depends only on the $j$-th coordinates of the peaks of the voters.

Lemma 5 Let $0<\gamma \leq 1$ and let $F$ be continuous, unanimous, and $\gamma-$ SP. Let $k \geq 2$, $j \in\{1, \ldots, k\}$, and let $R, R^{\prime} \in \rho$ with $R(t)=(p(t), w(t))$ and $R^{\prime}(t)=\left(p^{\prime}(t), w^{\prime}(t)\right)$ for each $t \in \Omega$ such that $p_{j}^{\prime}(t)=p_{j}(t)$ and $p_{\ell}^{\prime}(t)=0$ for all $t \in \Omega$ and $\ell \neq j$. Then $F_{j}\left(R^{\prime}\right)=F_{j}(R)$.

Proof W.1.o.g. assume $j=1$. Let $m$ be the smallest integer larger than or equal to $1 / \gamma$. Choose $\alpha>0$ arbitrary. Choose $\varepsilon>0$ arbitrary but with $\varepsilon<\alpha /(m+2)$. By Lemma 4 we can choose $\delta>0$ such that for all $p, x, y \in A$ with either $p_{1} \leq y_{1} \leq x_{1}-\varepsilon$ or $p_{1} \geq y_{1} \geq x_{1}+\varepsilon$ we have that a voter with preference $(p,(1, \delta, \ldots, \delta)) \in \mathcal{Q}$ strictly prefers $y$ to $x$. Let $Q$ be the profile with $Q(t)=\left(p^{\prime}(t),(1, \delta, \ldots, \delta)\right.$ for all $t \in \Omega$.

First consider the set of voters $S^{1}$ with $p_{1}(R(t)) \leq F_{1}(R(t))-\alpha$ for all $t \in S^{1}$. Let $\lambda\left(S^{1}\right)=\beta_{1}$ and let $m_{1}$ be the smallest integer larger than or equal to $\beta_{1} / \gamma$. By applying $\gamma$-SP $m_{1}$ times, each time changing the preferences of voters $t$ in a subset of $S^{1}$ of size at most $\gamma$ from $R(t)$ to $Q(t)$, we obtain for the profile $R^{1}$, defined by $R^{1}(t)=Q(t)$ for $t \in S^{1}$ and $R^{1}(t)=R(t)$ for $t \in \Omega \backslash S^{1}$, that $\left|F_{1}\left(R^{1}\right)-F_{1}(R)\right|<m_{1} \varepsilon$ by the choice of $\delta$.

Second, consider the set of voters $S^{2}$ with $p_{1}(R(t)) \geq F_{1}(R(t))+\alpha$ for all $t \in S^{2}$. Let $\lambda\left(S^{2}\right)=\beta_{2}$ and let $m_{2}$ be the smallest integer larger than or equal to $\beta_{2} / \gamma$. Repeat the above argument to obtain a profile $R^{2}$, defined by $R^{2}(t)=Q(t)$ for all $t \in S^{2} \cup S^{1}$ and $R^{2}(t)=R(t)$ for all $t \notin S^{2} \cup S^{1}$, and such that $\left|F_{1}\left(R^{2}\right)-F_{1}\left(R^{1}\right)\right|<m_{2} \varepsilon$, hence $\left|F_{1}\left(R^{2}\right)-F_{1}(R)\right|<\left(m_{1}+m_{2}\right) \varepsilon$. Hence, $F_{1}\left(R^{2}\right)$ is a number in the interval $\left(F_{1}(R)-\left(m_{1}+m_{2}\right) \varepsilon, F_{1}(R)+\left(m_{1}+m_{2}\right) \varepsilon\right)$.

Finally, change the preferences of the remaining voters $t$, those in $\Omega \backslash\left(S^{1} \cup S^{2}\right)$, from $R(t)$ to $Q(t)$. Let $m_{3}$ be the smallest integer larger than or equal to $\left(1-\beta_{1}-\beta_{2}\right) / \gamma$. By $\gamma$-SP it now follows that $F_{1}(Q)$ is a number in the interval $\left(F_{1}(R)-\left(m_{1}+\right.\right.$ $\left.\left.m_{2}+m_{3}\right) \varepsilon, F_{1}(R)+\left(m_{1}+m_{2}+m_{3}\right) \varepsilon\right)$. Since $m_{1}+m_{2}+m_{3} \leq m+2$, we have $\left|F_{1}(Q)-F_{1}(R)\right|<(m+2) \varepsilon<\alpha$.

By Lemma 3 we have $F\left(R^{\prime}\right)=F(Q)$, so in particular $\left|F_{1}\left(R^{\prime}\right)-F_{1}(R)\right|<$ $(m+2) \varepsilon<\alpha$. Since this holds for every $\alpha>0$ we conclude that $F_{1}(R)=F_{1}\left(R^{\prime}\right)$.

Proof of Theorem 2 Let $F$ be continuous, unanimous, and $\gamma$-SP. For each $j=1, \ldots, k$ define $F^{j}:[0,1]^{\Omega} \rightarrow[0,1]$ by $F^{j}\left((x(t))_{t \in \Omega}\right)=F\left(R^{x^{j}}\right)$ for each $(x(t))_{t \in \Omega} \in[0,1]^{\Omega}$, where $R^{x^{j}} \in \rho$ is some profile with $p_{j}\left(R^{x^{j}}(t)\right)=x(t)$ and 
$p_{\ell}\left(R^{x^{j}}(t)\right)=0$ for all $\ell \neq j$ and $t \in \Omega$. Then $F^{j}$ is well-defined because of Lemma 3, and $F^{j}$ inherits continuity, unanimity, and $\gamma$-SP from $F$. The proof of the theorem is complete by applying Lemma 5.

Open Access This article is distributed under the terms of the Creative Commons Attribution License which permits any use, distribution and reproduction in any medium, provided the original author(s) and source are credited.

\section{References}

Barberà S (2010) Strategy-proof social choice. Barcelona Economics Working Paper Series, No. 420

Barberà S, Berga D, Moreno B (2010) Individual versus group strategy-proofness: when do they coincide? J Econ Theory 145:1648-1674

Border KC, Jordan JS (1983) Straightforward elections, unanimity and phantom voters. Rev Econ Stud 50:153-170

Kirman A, Sondermann D (1972) Arrow's theorem, many agents, and invisible dictators. J Econ Theory 3:267-277

Maus S, Peters H, Storcken T (2006) Strategy-proof voting for single issues and cabinets. Public Choice 126:27-43

Moulin H (1980) On strategy-proofness and single-peakedness. Public Choice 35:437-455

Sprumont Y (1995) Strategyproof collective choice in economic and political environments. Can J Econ 28:68-107

Weymark J (1999) Decomposable strategy-proof social choice functions. Jpn Econ Rev 50:343-355

Zhou L (1991) Impossibility of strategy-proof mechanisms in economies with pure public goods. Rev Econ Stud 58:107-119 